NSF-KITP-14-116

\title{
On the Vlasov equation for Schwinger pair production in a time-dependent electric field
}

\author{
Adolfo Huet $^{a, b}$, Sang Pyo Kim ${ }^{c, d}$, Christian Schubert ${ }^{b, e}$ \\ a Departamento de Nanotecnología, Centro de Física Aplicada y Tec- \\ nología Avanzada, (CFATA) UNAM, Campus Juriquilla, Boulevard \\ Juriquilla No. 3001, C.P. 76230, A.P. 1-1010, Querétaro, México. \\ E-mail: ahuet@fata.unam.mx. \\ ${ }^{b}$ Instituto de Física y Matemáticas, Universidad Michoacana de San \\ Nicolás de Hidalgo, Edificio C-3, Apdo. Postal 2-82, C.P. 58040, \\ Morelia, Michoacán, México. E-mail: schubert@ifm.umich.mx. \\ c Department of Physics, Kunsan National University, Kunsan 573-701, \\ Korea.E-mail: sangkim@kunsan.ac.kr. \\ $d$ Center for Relativistic Laser Science, Institute for Basic Science, Gwangju \\ 500-712, Korea. \\ e Kavli Institute for Theoretical Physics, University of California, Santa \\ Barbara, CA 93106, USA.
}

\begin{abstract}
Schwinger pair creation in a purely time-dependent electric field can be described through a quantum Vlasov equation describing the time evolution of the single-particle momentum distribution function. This equation exists in two versions, both of which can be derived by a Bogoliubov transformation, but whose equivalence is not obvious. For the spinless case, we show here that the difference between these two evolution equations corresponds to the one between the "in-out" and "in-in" formalisms. We give a simple relation between the asymptotic distribution functions generated by the two Vlasov equations. As examples we discuss the Sauter and single-soliton field cases.
\end{abstract}




\section{Introduction}

The QED effect of spontaneous pair creation of electron-positron pairs by a strong electric field was conceived by Sauter as early as 1931 [1] and computed by Schwinger in 1951 for the constant field case [2]. Considerable work has gone into developing techniques for the calculation of this effect for more realistic electric field configurations [3, 4, 15, 6, 7, , 8, 9, 10, 11, 12, 13, 14, 15, in particular such as can be realized by laser fields (see, e.g., [16, 17, 18]). In this effort, a special role is played by the case of a purely time-dependent field. This is because, although even here analytic results cannot be expected in the generic case, a formalism exists that allows one to compute the pair creation rate numerically in a straightforward manner. What makes the purely time-dependent case very special is that for such a field the spatial momentum $\mathbf{k}$ is a good quantum number. Thus one can fix it, and use a Bogoliubov transformation between the vacua at initial time and time $t$ to derive an evolution equation for the density of pairs $\mathcal{N}_{\mathbf{k}}(t)$ with fixed momenta. This is the quantum Vlasov equation ('QVE'). In the scalar QED case, which we will consider in this paper, the QVE is usually written in the form $7,19,19,20,21,22,23,24,25]$

$$
\begin{aligned}
\dot{\mathcal{N}}_{\mathbf{k}}(t)= & \frac{\dot{\omega}_{\mathbf{k}}(t)}{2 \omega_{\mathbf{k}}(t)} \int_{-\infty}^{t} d t^{\prime} \frac{\dot{\omega}_{\mathbf{k}}\left(t^{\prime}\right)}{\omega_{\mathbf{k}}\left(t^{\prime}\right)}\left(1+2 \mathcal{N}_{\mathbf{k}}\left(t^{\prime}\right)\right) \\
& \times \cos \left[2 \int_{t^{\prime}}^{t} d t^{\prime \prime} \omega_{\mathbf{k}}\left(t^{\prime \prime}\right)\right]
\end{aligned}
$$

where $\omega_{\mathbf{k}}^{2}(t)$ is the total energy squared,

$$
\omega_{\mathbf{k}}^{2}(t)=\left(k_{\|}-q A_{\|}(t)\right)^{2}+\mathbf{k}_{\perp}^{2}+m^{2} .
$$

Here the temporal gauge $A_{0}=0, \dot{\mathbf{A}}(t)=-\mathbf{E}(t)$ has been used. The field points into a fixed direction, and $k_{\|}$denotes the canonical three-momentum component along the field. $\mathcal{N}_{\mathbf{k}}(t)$ is usually taken to be zero initially, that is at $t=-\infty$, and for $t \rightarrow \infty$ turns into the density of created pairs with fixed momentum $\mathbf{k}$. This interpretation has been shown to be consistent with various other approaches to the time-dependent Schwinger problem, such as the one-loop effective action [19], the quantum mechanical scattering approach [25] and the Wigner formalism [26, 27, 28, 29]. From a practical point of view, although the structure of (1) is not promising for attempts at exact analytical solution, it is amenable to numerical evaluation, as well 
as to other approximation schemes [19, 22, 23, 24, 25]. Sometimes it is preferable to use the following equivalent system of first-order differential equations [23]

$$
\frac{d}{d t}\left(\begin{array}{c}
1+2 \mathcal{N}_{\mathbf{k}} \\
\mathcal{M}_{\mathbf{k}}^{(-)} \\
\mathcal{M}_{\mathbf{k}}^{(+)}
\end{array}\right)=\left(\begin{array}{ccc}
0 & \frac{\dot{\omega}_{\mathbf{k}}}{\omega_{\mathbf{k}}} & 0 \\
\frac{\dot{\omega}_{\mathbf{k}}}{\omega_{\mathbf{k}}} & 0 & -2 \omega_{\mathbf{k}} \\
0 & 2 \omega_{\mathbf{k}} & 0
\end{array}\right)\left(\begin{array}{c}
1+2 \mathcal{N}_{\mathbf{k}} \\
\mathcal{M}_{\mathbf{k}}^{(-)} \\
\mathcal{M}_{\mathbf{k}}^{(+)}
\end{array}\right)
$$

where $\mathcal{M}_{\mathrm{k}}^{( \pm)}$are two auxiliary functions.

As has been emphasized in [19, 30], no direct physical meaning should be ascribed to $\mathcal{N}_{\mathbf{k}}(t)$ at intermediate times, due to its dependence on the choice of an instantaneous adiabatic basis. This implies a large ambiguity which one can try to use for the construction of simpler, but physically equivalent evolution equations. In [31] two of the authors obtained, also using a Bogoliubov transformation but in a form inspired by Lewis-Riesenfeld theory [32, 33, 34, 35], the following alternative evolution equation (see also the recent [36] for a systematic approach to the construction of a mathematically optimized adiabatic basis)

$$
\begin{aligned}
\dot{\tilde{\mathcal{N}}}_{\mathbf{k}}(t)= & \frac{1}{2} \Omega_{\mathbf{k}}^{(-)}(t) \int_{t_{0}}^{t} d t^{\prime}\left[\Omega_{\mathbf{k}}^{(-)}\left(t^{\prime}\right)\left(1+2 \tilde{\mathcal{N}}_{\mathbf{k}}\left(t^{\prime}\right)\right)\right. \\
& \left.\times \cos \left(\int_{t^{\prime}}^{t} d t^{\prime \prime} \Omega_{\mathbf{k}}^{(+)}\left(t^{\prime \prime}\right)\right)\right]
\end{aligned}
$$

Here $\tilde{\mathcal{N}}_{\mathbf{k}}(t)=0$ at $t=-\infty$ as before, and

$$
\Omega_{\mathbf{k}}^{( \pm)}(t):=\frac{\omega_{\mathbf{k}}^{2}(t) \pm \omega_{\mathbf{k} i}^{2}}{\omega_{\mathbf{k} i}}
$$

where

$$
\omega_{\mathbf{k} i}:=\lim _{t \rightarrow-\infty} \omega_{\mathbf{k}}(t)
$$

Thus we have to assume in the following that this limit is finite, and similarly also 


$$
\omega_{\mathbf{k} f}:=\lim _{t \rightarrow+\infty} \omega_{\mathbf{k}}(t)
$$

However, this is a minor restriction since, for electric fields that vanish sufficiently fast at $t= \pm \infty$, it can always be achieved in a suitable gauge.

Note that, in contrast to the coefficient functions appearing in the "standard" Vlasov equation (1), the coefficient functions $\Omega_{\mathbf{k}}^{( \pm)}(t)$ do not involve square roots of time-dependent quantities.

For the "alternative" Vlasov equation, two equivalent differential equations were obtained in [31]. The first one is a system of first-order differential equations analogous to (3),

$$
\frac{d}{d t}\left(\begin{array}{c}
1+2 \tilde{\mathcal{N}}_{k} \\
\tilde{\mathcal{M}}_{k}^{(-)} \\
\tilde{\mathcal{M}}_{k}^{(+)}
\end{array}\right)=\left(\begin{array}{ccc}
0 & \Omega_{k}^{(-)} & 0 \\
\Omega_{k}^{(-)} & 0 & \Omega_{k}^{(+)} \\
0 & -\Omega_{k}^{(+)} & 0
\end{array}\right)\left(\begin{array}{c}
1+2 \tilde{\mathcal{N}}_{k} \\
\tilde{\mathcal{M}}_{k}^{(-)} \\
\tilde{\mathcal{M}}_{k}^{(+)}
\end{array}\right)
$$

The second one is the following linear third order linear differential equation

$$
\dddot{F}+4 \omega^{2} \dot{F}+2\left(\omega^{2}\right)^{\cdot} F=\frac{\left(\omega^{2}\right)^{\cdot}}{\omega_{i}^{2}}
$$

where the relation between $F(t)$ and $\tilde{\mathcal{N}}(t)$ is given by (here and in the following we often drop the subscript $\mathbf{k}$ )

$$
\tilde{\mathcal{N}}(t)=\frac{\omega_{i}}{2} \int_{-\infty}^{t} d t^{\prime} \dot{F}\left(t^{\prime}\right) \Omega^{(-)}\left(t^{\prime}\right)
$$

(our $\omega_{i}$ corresponds to $\omega_{0}$ in [31]).

For the alternative Vlasov equation, an interesting set of explicit solutions could be found in terms of the well-known solitons of the Korteweg-de Vries equation [31, 37, 38].

Mathematically the two evolution equations (1) and (4) are clearly not equivalent, and it remained an open problem to elucidate the physical relation between them. The purpose of the present paper is to show that the difference between them corresponds precisely to the one between the "inin" and "in-out" formalisms. This will also allow us to find a simple relation 
between the asymptotic distribution functions $\mathcal{N}(t=\infty)$ and $\tilde{\mathcal{N}}(t=\infty)$. We carry this through in chapters 2 and 3 . As examples, in chapter 4 we discuss the Sauter and single-soliton field cases.

\section{The in-out Vlasov equation}

In this chapter we will verify explicitly that the "standard" Vlasov equation corresponds to the "in-out" formalism. The argument essentially follows [30]. The basic equation underlying both evolutions equations is the classical mode equation

$$
\ddot{\phi}(t)+\omega^{2}(t) \phi(t)=0 .
$$

Assuming that $\phi$ fulfills the mode equation (11), then

$$
\mathcal{N}(t):=\frac{|\dot{\phi}(t)|^{2}+\omega^{2}(t)|\phi(t)|^{2}}{2 \omega(t)}-\frac{1}{2}
$$

solves the Vlasov equation (1). This can be verified most directly by plugging $\mathcal{N}(t)$ into the equivalent differential equation (3), where the two auxiliary functions $\mathcal{M}^{( \pm)}$are given by

$$
\begin{aligned}
\mathcal{M}^{(+)}(t) & =-\frac{d}{d t}|\phi(t)|^{2} \\
\mathcal{M}^{(-)}(t) & =-\frac{|\dot{\phi}(t)|^{2}-\omega^{2}(t)|\phi(t)|^{2}}{\omega(t)} .
\end{aligned}
$$

Now, under our assumption that $\omega_{i}:=\lim _{t \rightarrow-\infty} \omega(t)$ converges, we can decompose the mode solution for $t \rightarrow-\infty$ as

$$
\phi(t) \stackrel{t \rightarrow-\infty}{\longrightarrow} A_{0} \frac{\mathrm{e}^{i \omega_{i} t}}{\sqrt{2 \omega_{i}}}+B_{0} \frac{\mathrm{e}^{-i \omega_{i} t}}{\sqrt{2 \omega_{i}}}
$$

leading to

$$
\mathcal{N}(t) \stackrel{t \rightarrow-\infty}{\longrightarrow} \frac{1}{2}\left(\left|A_{0}\right|^{2}+\left|B_{0}\right|^{2}\right)-\frac{1}{2} .
$$


If we further assume that initially there are no particles present, standard quantum field theory arguments imply that $A_{0}=0$, and $\lim _{t \rightarrow-\infty} \mathcal{N}(t)=0$ together with (15) then shows that $\left|B_{0}\right|^{2}=1$. Thus the relevant solution of the mode equation will obey (up to an irrelevant phase factor)

$$
\phi(t) \stackrel{t \rightarrow-\infty}{\longrightarrow} \frac{1}{\sqrt{2 \omega_{i}}} \mathrm{e}^{-i \omega_{i} t}
$$

At future infinity, in the in-out formalism we have to expand the mode solution in terms of plane waves with the final frequency,

$$
\phi(t) \stackrel{t \rightarrow+\infty}{\longrightarrow} A_{\infty} \frac{\mathrm{e}^{i \omega_{f} t}}{\sqrt{2 \omega_{f}}}+B_{\infty} \frac{\mathrm{e}^{-i \omega_{f} t}}{\sqrt{2 \omega_{f}}} .
$$

Similarly to 15 this yields

$$
\mathcal{N}_{\infty}:=\lim _{t \rightarrow \infty} \mathcal{N}(t)=\frac{1}{2}\left(\left|A_{\infty}\right|^{2}+\left|B_{\infty}\right|^{2}\right)-\frac{1}{2}
$$

A further relation is obtained from the Wronskian constraint (see, e.g., [31])

$$
\mathrm{Wr}\left[\phi, \phi^{*}\right] \equiv \phi(t) \dot{\phi}^{*}(t)-\phi^{*}(t) \dot{\phi}(t)=i
$$

which gives

$$
\left|B_{\infty}\right|^{2}-\left|A_{\infty}\right|^{2}=1
$$

Using this equation in 18 to eliminate $B_{\infty}$ we obtain the simple expression

$$
\mathcal{N}_{\infty}=\left|A_{\infty}\right|^{2}
$$

for the final density of created pairs (at fixed momentum $\mathbf{k}$ ). 


\section{The in-in Vlasov equation}

We will now proceed analogously for the alternative Vlasov equation (4). Here the analogue of (12), that is, an explicit solution of (4) in terms of the solution of the mode equation (11), turns out to be

$$
\tilde{\mathcal{N}}(t)=\frac{|\dot{\phi}(t)|^{2}+\omega_{i}^{2}|\phi(t)|^{2}}{2 \omega_{i}}-\frac{1}{2}
$$

as can again be verified simply by plugging into the differential equation system (8). The auxiliary functions $\tilde{\mathcal{M}}^{( \pm)}$are now given by

$$
\begin{aligned}
\tilde{\mathcal{M}}^{(+)} & =-\frac{|\dot{\phi}(t)|^{2}-\omega_{i}^{2}|\phi(t)|^{2}}{\omega_{i}} \\
\tilde{\mathcal{M}}^{(-)} & =-\frac{d}{d t}|\phi(t)|^{2}
\end{aligned}
$$

(note the interchange of $(+)$ and $(-)$ as compared to (13)). The corresponding solution of the third-order differential equation (9) is

$$
F(t)=-\frac{1}{\omega_{i}}\left(|\phi(t)|^{2}-\frac{1}{2 \omega_{i}}\right) .
$$

For the sake of completeness, in the appendix we show how to derive this solution, as well as the equation itself, directly from Lewis-Riesenfeld theory.

Since (22) differs from (12) only by the replacement of $\omega(t)$ by $\omega_{i}$, the asymptotic expansion of $\tilde{\mathcal{N}}(t)$ for $t \rightarrow-\infty$ agrees with the one of $\mathcal{N}(t)$, eq. (15). However, at large times things are very different. To get formulas analogous to (17), 18 we now have to project on plane waves with the initial frequencies. Namely, defining $\tilde{A}_{\infty}, \tilde{B}_{\infty}$ by

$$
\phi(t) \stackrel{t \rightarrow+\infty}{\longrightarrow} \tilde{A}_{\infty} \frac{\mathrm{e}^{i \omega_{i} t}}{\sqrt{2 \omega_{i}}}+\tilde{B}_{\infty} \frac{\mathrm{e}^{-i \omega_{i} t}}{\sqrt{2 \omega_{i}}}
$$

eq. 22 gives

$$
\tilde{\mathcal{N}}(t) \stackrel{t \rightarrow+\infty}{\longrightarrow} \frac{1}{2}\left(\left|\tilde{A}_{\infty}\right|^{2}+\left|\tilde{B}_{\infty}\right|^{2}\right)-\frac{1}{2}
$$


Using also the Wronskian constraint to eliminate $\tilde{B}_{\infty}$, we find

$$
\tilde{\mathcal{N}}_{\infty}:=\lim _{t \rightarrow \infty} \tilde{\mathcal{N}}(t)=\left|\tilde{A}_{\infty}\right|^{2}
$$

Thus $\tilde{\mathcal{N}}$ asymptotically measures the presence of negative frequency components with respect to the initial energies. This provides an explicit way of seeing that the alternative Vlasov equation (4) pertains to the in-in formalism.

To be able to transform between the two equations we need to know also how $\tilde{\mathcal{N}}(t)$ is written asymptotically in terms of the coefficients $A_{\infty}, B_{\infty}$ of the expansion of $\phi$ in terms of final frequencies, eq. (17). Here we find

$$
\begin{aligned}
& \tilde{\mathcal{N}}(t) \stackrel{t \rightarrow+\infty}{\longrightarrow} \frac{\omega_{i}^{2}+\omega_{f}^{2}}{4 \omega_{i} \omega_{f}}\left(\left|A_{\infty}\right|^{2}+\left|B_{\infty}\right|^{2}\right)-\frac{1}{2} \\
&+\frac{\omega_{i}^{2}-\omega_{f}^{2}}{4 \omega_{i} \omega_{f}}\left(A_{\infty} B_{\infty}^{*} \mathrm{e}^{2 i \omega_{f} t}+A_{\infty}^{*} B_{\infty} \mathrm{e}^{-2 i \omega_{f} t}\right) .
\end{aligned}
$$

Comparing with 18 we see that $\mathcal{N}(t)$ and $\tilde{\mathcal{N}}(t)$ will agree for $t \rightarrow \infty$ if and only if $\omega_{f}=\omega_{i}$. Otherwise $\tilde{\mathcal{N}}(t)$ will not even converge, due to the oscillating terms in the second line of (28). To eliminate these terms, we can use asymptotic averaging. Defining the asymptotic average of a function $f(t)$ for large $t$ as usual by

$$
\langle f\rangle_{\infty}:=\lim _{t \rightarrow \infty} \frac{1}{t} \int_{t}^{2 t} d t^{\prime} f\left(t^{\prime}\right)
$$

we get the transformation formula

$$
2 \mathcal{N}_{\infty}+1=2 \frac{\omega_{i} \omega_{f}}{\omega_{i}^{2}+\omega_{f}^{2}}\langle 2 \tilde{\mathcal{N}}+1\rangle_{\infty}
$$

\section{Examples}

We will illustrate the differences between $\mathcal{N}(t)$ and $\tilde{\mathcal{N}}(t)$ with two examples for which closed-form solutions of the mode equation (11) exist, namely the 
"time-like Sauter field" and the "single-soliton field". The time-like Sauter field is defined by

$$
E(t)=E_{0} \operatorname{sech}^{2}(t / \tau)
$$

which we can realize by

$$
A^{\mu}=\left(0,0,0, E_{0} \tau(1+\tanh (t / \tau))\right) .
$$

The solution to the mode equation obeying (16) is [39]

$$
\begin{aligned}
& \phi(t)=\frac{1}{\sqrt{2 \omega_{0} \mathrm{e}^{\pi \omega_{o} \tau}}}\left(-\mathrm{e}^{\frac{2 t}{\tau}}\right)^{-\frac{i}{2} \omega_{0} \tau}\left(1+\mathrm{e}^{\frac{2 t}{\tau}}\right)^{\frac{1}{2}+i \sqrt{-\frac{1}{4}+E_{0}^{2} \tau^{4}}} \\
& \times_{2} F_{1}\left(\frac{1}{2}-\frac{i}{2}\left(\omega_{0} \tau-2 \sqrt{-\frac{1}{4}+E_{0}^{2} \tau^{4}}-\tau \sqrt{\omega_{0}^{2}-4 E_{0} \tau+4 E_{0}^{2} \tau^{2}}\right),\right. \\
& \left.\frac{1}{2}-\frac{i}{2}\left(\omega_{0} \tau-2 \sqrt{-\frac{1}{4}+E_{0}^{2} \tau^{4}}+\tau \sqrt{\omega_{0}^{2}-4 E_{0} \tau+4 E_{0}^{2} \tau^{2}}\right), 1-i \omega_{0} \tau,-\mathrm{e}^{\frac{2 t}{\tau}}\right) .
\end{aligned}
$$

where $\omega_{0} \equiv \sqrt{\mathbf{k}^{2}+m^{2}}$, and the gauge has been chosen such that $\omega_{i}=\omega_{0}$. For our example, we use the parameters $E_{0}=q=k_{\|}=1, \omega_{0}=1.1, \tau=2$. Plugging $\phi(t)$ into 12 and 22 results in expressions for $\mathcal{N}(t), \tilde{\mathcal{N}}(t)$ which are too lengthy to be given here. A MATHEMATICA plot of both function for the our parameter values is shown in fig. 1. Here $\omega_{f}=3.04 \neq \omega_{i}=$ $\omega_{0}=1.1$, therefore as stated above $\tilde{\mathcal{N}}(t)$ keeps oscillating for $t \rightarrow \infty$. It is easily checked that the transformation formula 30 between $\mathcal{N}_{\infty}$ and $\langle\tilde{\mathcal{N}}\rangle_{\infty}$ is fulfilled.

Our second example is a "solitonic" electric field. In [31, 37] it had been shown that an infinite set of special solutions to the alternative Vlasov equation can be explicitly constructed using the well-known soliton solutions of the Korteweg-de-Vries equation. The simplest one of these field configurations is given by

$$
q A(t)=k_{\|}-\sqrt{k_{\|}^{2}+\frac{2 \omega_{0}^{2}}{\cosh ^{2}\left(\omega_{0} t\right)}}
$$

where again $\omega_{i}=\omega_{0}$. The solitonic character of the associated solutions shows itself in the property that $\lim _{t \rightarrow \infty} \mathcal{N}(t)=0$ for the $\mathbf{k}$ used in the 


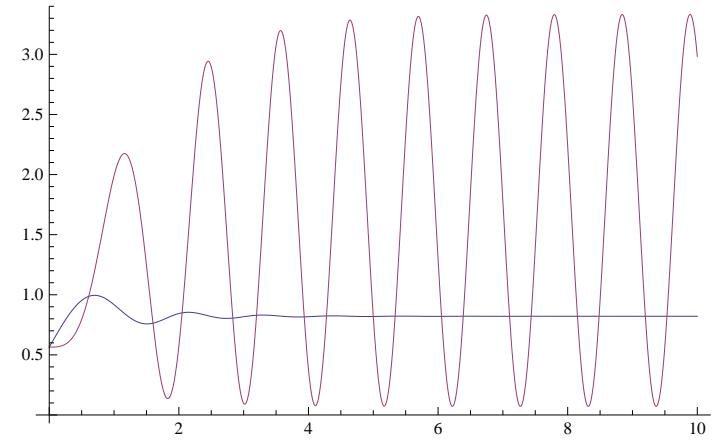

Figure 1: A comparison of $\mathcal{N}(t)$ (damped oscillating blue curve) against $\tilde{\mathcal{N}}(t)$ (oscillating red curve) for the timelike Sauter field.

definition of the field (34). This does not mean that such an electric field does not pair-create at all, but it can be tuned not to pair-create at a given momentum. The solution to the mode equation obeying (16) is [31]

$$
\phi(t)=\frac{1}{\sqrt{2 \omega_{0}}} \frac{\mathrm{e}^{-i \omega_{0} t}\left(1-i \mathrm{e}^{2 \omega_{0} t}\right)}{1+\mathrm{e}^{2 \omega_{0} t}} .
$$

Plugging this into 12 reps. 22 gives

$$
\begin{aligned}
& \mathcal{N}(t)=\frac{4+\operatorname{sech}^{4}\left(\omega_{0} t\right)\left(1+2 \cosh \left(2 \omega_{0} t\right)\right)}{8 \sqrt{1+2 \operatorname{sech}^{2}\left(\omega_{0} t\right)}}-\frac{1}{2}, \\
& \tilde{\mathcal{N}}(t)=\frac{1}{8 \cosh ^{4}\left(\omega_{0} t\right)} .
\end{aligned}
$$

In fig. 2 we show a plot of both functions $\mathcal{N}(t), \tilde{\mathcal{N}}(t)$. Note the simplicity of $\tilde{\mathcal{N}}(t)$, the symmetry with respect to $t=0$, the absence of oscillations and the vanishing of both functions for $t \rightarrow \infty$.

\section{Conclusions}

We have clarified here the relation of the Vlasov equation for the creation of scalar pairs by time-dependent fields, recently proposed in [31], to the older 


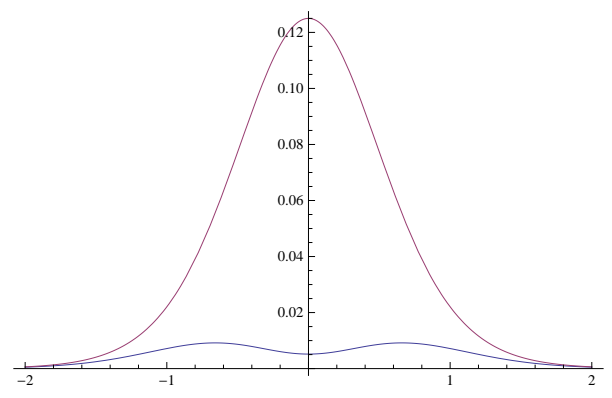

Figure 2: A comparison of $\mathcal{N}(t)$ (double peaked blue curve) against $\tilde{\mathcal{N}}(t)$ (single peaked red curve) for the simplest solitonic field.

and widely used one of [9]. The difference between both equations boils down to a projection on final states with initial vs. final frequencies, that is, just to the distinction between the "in-in" vs. the "in-out" formalisms. If the initial and final frequencies are equal, $\omega_{f}=\omega_{i}$, the asymptotic pair production rates coincide. This is the case, in particular, for the family of "solitonic" fields found in [31, 37]. Thus the property of the associated solutions of the alternative Vlasov equation to have a vanishing asymptotic pair creation rate is shared by the corresponding solutions of the standard Vlasov equation.

If $\omega_{f} \neq \omega_{i}$ the latter equation is, as the in-out one, the appropriate one in the context of laboratory experiments (the in-in formalism figures prominently in cosmology, see, e.g., [40, 41]). In any case, with the transformation formula (30) in hand both equations can be used equivalently according to mathematical convenience.

Acknowledgements: The authors would like to thank R. Alkofer, G.V. Dunne, A. M. Fedotov, H. Gies, F. Hebenstreit and S.-J. Rey for discussions and correspondence. C. S. thanks the KITP for hospitality during the program 'Frontiers of intense laser physics', as well as the Physics Department of Kunsan National University, Korea, and the Center for Theoretical Physics, Seoul National University. The work of A. Huet and C. Schubert was supported by CONACYT, Mexico, the work of S. P. K. by the Research Center Program of IBS (Institute for Basic Science, Korea) under IBS-R012-D1, and the work of C. S. by the NSF under Grant No. NSF PHY11-25915. 


\section{Appendix}

For completeness, in this appendix we provide a direct derivation of the third-order equation (9), and its solution, using the Lewis-Riesenfeld approach to QED of 31]. To avoid undue repetition, in this appendix we refer the reader to [31] for the general set-up and notation. The Hermitian conjugates of the fields in (6) of [31] are

$$
\begin{aligned}
& \hat{\phi}_{\mathbf{k}}^{\dagger}=\hat{a}_{\mathbf{k}}^{\dagger}(t) \phi_{\mathbf{k}}^{*}(t)+\hat{b}_{-\mathbf{k}}(t) \phi_{\mathbf{k}}(t), \\
& \hat{\pi}_{\mathbf{k}}^{\dagger}=\hat{a}_{\mathbf{k}}(t) \dot{\phi}_{\mathbf{k}}(t)+\hat{b}_{-\mathbf{k}}^{\dagger}(t) \dot{\phi}_{\mathbf{k}}^{*}(t),
\end{aligned}
$$

where the quantum invariants $\hat{a}_{\mathbf{k}}(t), \hat{a}_{\mathbf{k}}^{\dagger}(t)$ and $\hat{b}_{-\mathbf{k}}(t), \hat{b}_{-\mathbf{k}}^{\dagger}(t)$ are the timedependent annihilation and creation operators for particle and antiparticle, respectively, and the auxiliary field $\phi_{\mathbf{k}}$ obeys (11). The vacuum expectation values of the equal-time correlation functions are

$$
\begin{aligned}
\left\langle\hat{\phi}_{\mathbf{k}}^{\dagger} \hat{\phi}_{\mathbf{k}}\right\rangle & =\left|\phi_{\mathbf{k}}(t)\right|^{2}, \\
\left\langle\hat{\pi}_{\mathbf{k}}^{\dagger} \hat{\phi}_{\mathbf{k}}^{\dagger}+\hat{\phi}_{\mathbf{k}} \hat{\pi}_{\mathbf{k}}\right\rangle & =\frac{d}{d t}\left|\phi_{\mathbf{k}}(t)\right|^{2}, \\
\left\langle\hat{\pi}_{\mathbf{k}}^{\dagger} \hat{\pi}_{\mathbf{k}}\right\rangle & =\left|\dot{\phi}_{\mathbf{k}}(t)\right|^{2} .
\end{aligned}
$$

The mode solution may be written as (now dropping the subscript $\mathbf{k}$ )

$$
\phi(t)=\rho(t) e^{-i \theta(t)},
$$

which from the quantization rule (19) satisfies the nonlinear equation

$$
\ddot{\rho}(t)+\omega^{2}(t) \rho(t)-\frac{1}{4 \rho^{3}(t)}=0 .
$$

The expectation value of the Hamiltonian (1) of [31] equated to the integral of the frequency change for the potential energy

$$
\langle\hat{H}(t)\rangle=\dot{\rho}^{2}+\omega^{2} \rho^{2}+\frac{1}{4 \rho^{2}}=\int^{t} d t^{\prime}\left(\omega^{2}\right)^{\cdot} \rho^{2}
$$

leads to 40$)$. We may write the expectation values $(38)$ in terms of $\rho$ :

$$
\begin{aligned}
\left\langle\hat{\phi}^{\dagger} \hat{\phi}\right\rangle & =\rho^{2}, \\
\left\langle\hat{\pi}^{\dagger} \hat{\phi}^{\dagger}+\hat{\phi} \hat{\pi}\right\rangle & =2 \rho \dot{\rho}, \\
\left\langle\hat{\pi}^{\dagger} \hat{\pi}\right\rangle & =\dot{\rho}^{2}+\frac{1}{4 \rho^{2}} .
\end{aligned}
$$


Finally, using (39) and 40 we obtain $(24)$ and its first three derivatives

$$
\begin{aligned}
\omega_{i} F & =-\rho^{2}+\frac{1}{2 \omega_{i}}, \\
\omega_{i} \dot{F} & =-2 \rho \dot{\rho}, \\
\omega_{i} \ddot{F} & =-2\left(\dot{\rho}^{2}+\frac{1}{4 \rho^{2}}\right)+2 \omega^{2} \rho^{2}, \\
\omega_{i} \dddot{F} & =8 \omega^{2} \rho \dot{\rho}+2\left(\omega^{2}\right)^{\cdot} \rho^{2} .
\end{aligned}
$$

Now $(9$ follows from 43 . Note that $F, \dot{F}$ and $\ddot{F}$ are determined by the correlation functions $(42)$ and that $(9)$ is a linearization of (40).

\section{References}

[1] F. Sauter, Z. Phys. 69, 742 (1931).

[2] J. Schwinger, Phys. Rev. 82, 664 (1951).

[3] E. Brézin and C. Itzykson, Phys. Rev. D 2, 1191 (1970).

[4] V. S. Popov, Sov. Phys. JETP 34, 709 (1972); 35, 659 (1972).

[5] V. G. Bagrov, D. M. Gitman, S. P. Gavrilov and Sh. M. Shvartsman, Izv. Vuz. Fiz. 3, 71 (1975) [Sov. Phys. J. 18, 351 (1975)].

[6] M. S. Marinov and V. S. Popov, Fortschr. Phys. 25, 373 (1977).

[7] Y. Kluger, J. M. Eisenberg, B. Svetitsky, F. Cooper and E. Mottola, Phys. Rev. Lett. 67, 2427 (1991); Phys. Rev. D 45, 4659 (1992).

[8] S. P. Gavrilov and D. M. Gitman, Phys. Rev. D 53, 7162 (1996), hepth/9603152.

[9] Y. Kluger, E. Mottola and J. M. Eisenberg, Phys. Rev. D 58, 125015 (1998).

[10] S. P. Kim and D. N. Page, Phys. Rev. D 65, 105002 (2002), hepth/0005078; Phys. Rev. D 73, 065020 (2006), hep-th/0301132; Phys. Rev. D 75, 045013 (2007), hep-th/0701047.

[11] A. Di Piazza, Phys. Rev. D 70, 053013 (2004). 
[12] H. Gies and K. Klingmüller, Phys. Rev. D 72, 065001 (2005), hep$\mathrm{ph} / 0505099$.

[13] G. V. Dunne and C. Schubert, Phys. Rev. D 72, 105004 (2005), arXiv:hep-th/0507174.

[14] G. V. Dunne, Q.-h. Wang, H. Gies and C. Schubert, Phys. Rev. D 73, 065028 (2006), hep-th/0602176.

[15] C. K. Dumlu and G. V. Dunne, Phys. Rev. Lett. 104, 250402 (2010), arXiv:1004.2509; Phys. Rev. D 83, 065028 (2011), arXiv:1102.2899.

[16] A. Ringwald, Phys. Lett. B 510, 107 (2001), hep-ph/0103185.

[17] G. V. Dunne, Eur. Phys. J. D 55, 327 (2009), arXiv:0812.3163.

[18] T. Tajima, Eur. Phys. J. D 55, 519 (2009).

[19] F. Hebenstreit, R. Alkofer and H. Gies, Phys. Rev. D 78, 061701 (2008), arXiv:0807.2785.

[20] S.A. Smolyansky, G. Röpke, S.M. Schmidt, D. Blaschke, V.D. Toneev, and A.V. Prozorkevich, hep-ph/9712377.

[21] S. Schmidt, D. Blaschke, G. Röpke, S. A. Smolyansky, A. V. Prozorkevich and V. D. Toneev, Int. J. Mod. Phys. E 7 (1998) 709.

[22] S. Schmidt, D. Blaschke, G. Röpke, A. V. Prozorkevich, S. A. Smolyansky and V. D. Toneev, Phys. Rev. D 59, 094005 (1999), hep$\mathrm{ph} / 9810452$.

[23] J. C. Bloch, V. A. Mizerny, A. V. Prozorkevich, C. D. Roberts, S. M. Schmidt, S. A. Smolyansky and D. V. Vinnik, Phys. Rev. D 60116011 (1999), nucl-th/9907027

[24] R. Alkofer, M. B. Hecht, C. D. Roberts, S. M. Schmidt and D. V. Vinnik, Phys. Rev. Lett. 87193902 (2001), nucl-th/0108046.

[25] C. K. Dumlu, Phys. Rev. D 79 (2009) 065027, arXiv:0901.2972 [hep-th].

[26] S. R. deGroot, W. A. van Leeuwen and C. G. van Weert, Relativistic Kinetic Theory, North-Holland 1980.

[27] H.-T. Elze and U. Heinz, Phys. Rep. 183, 81 (1989). 
[28] I. Bialynicki-Birula, P. Górnicki and J. Rafelski, Phys. Rev. D 44, 1825 (1991).

[29] F. Hebenstreit, R. Alkofer and H. Gies, Phys. Rev. D 82, 105026 (2010), arXiv:1007.1099 [hep-ph].

[30] A. M. Fedotov, E. G. Gelfer, K.Yu. Korolev and S. A. Smolyansky, Phys. Rev. D 83, 025011 (2011), arXiv:1008.2098 [hep-ph].

[31] S. P. Kim and C. Schubert, Phys. Rev. D 84, 125028 (2011), arXiv:1110.0900 [hep-th].

[32] H. R. Lewis and W. B. Riesenfeld, J. Math. Phys. 10, 1458 (1969).

[33] I. A. Malkin, V. I. Man'ko and D. A. Trifonov, Phys. Rev. D 2, 1371 (1970); J. Math. Phys. 14, 576 (1973).

[34] F. Cooper, J. F. Dawson, D. Meredith and H. Shepard, Phys. Rev. Lett. 72, 1337 (1994).

[35] L. Faccioli, F. Finelli, G. P. Vacca and G. Venturi, Phys. Rev. Lett. 81, 240 (1998).

[36] R. Dabrowski and G.V. Dunne, Phys. Rev. D 90, 025021 (2014), arXiv:1405.0302 [hep-th].

[37] S. P. Kim, "Schwinger Pair Production in Solitonic Gauge Fields", arXiv:1110.4684 [hep-th].

[38] A. Huet, S. P. Kim and C. Schubert, Proc. of LPHYS 13, J. Phys.: Conf. Ser. 497 (2014) 012039.

[39] S. P. Kim, H. K. Lee and Y. Yoon, Phys. Rev. D 78, 105013 (2008), arXiv:0807.2696 [hep-th].

[40] A. Higuchi, D. Marolf and I. A. Morrison, Phys. Rev. D. 83, 084029 (2011), arXiv:1012.3415 [gr-qc].

[41] I. G. Moss, "Anomalies, boundaries and the in-in formalism", arXiv:1201.5732 [hep-th]. 